\title{
Chefarzt macht Krankenhaus Konkurrenz Fristlose Kündigung berechtigt!
}

Das Landesarbeitsgericht (LAG) Baden-Württemberg bestätigte die fristlose Kündigung eines Chefarztes durch das Krankenhaus ohne vorherige Abmahnung. Der Chefarzt hatte als Vertragsarzt massive Wettbewerbsverstöße begangen, die es dem Krankenhaus unzumutbar erscheinen ließen, das Arbeitsverhältnis bis zum Ablauf der Frist für eine ordentliche Kündigung fortzusetzen.

\section{Der Fall}

Ein 64-jähriger angestellter Chefarzt im Fachbereich Pathologie hatte mit der Klinik einen Nutzungs- und Kooperationsvertrag geschlossen, der es ihm ermöglichte, mit 50\% seiner Arbeitskraft eine Vertragsarztpraxis zu betreiben, die in einem räumlich getrennten Bereich auf dem Gelände der Klinik eingerichtet war. Aufgrund dieses Vertrages war es dem Chefarzt gestattet, auch externe Gewebeproben von ambulanten Leistungserbringern in seiner Vertragsarztpraxis zu begutachten und abzurechnen und nicht im Institut für Pathologie der Klinik. Dort wurden die stationär entnommenen Gewebeproben externer Leistungserbringer unter der Leitung des Chefarztes begutachtet und von der Klinik abgerechnet. Der Chefarzt verlegte die von ihm betriebene Vertragsarztpraxis später in ein Gebäude außerhalb des Klinikums und kün-

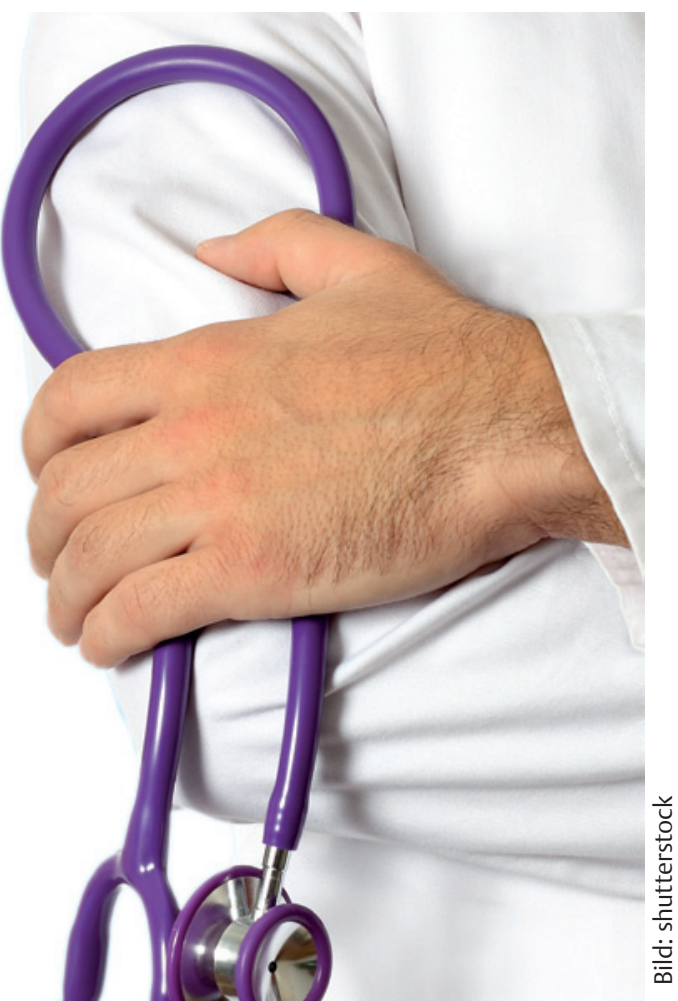

digte den bestehenden Nutzungs- und Kooperationsvertrag teilweise, nämlich nur soweit er den Betrieb der Vertragsarztpraxis auf dem Klinikgelände beinhaltete. Diese Teilkündigung wurde von der Klinik als unzulässig erachtet. Der Chefarzt wurde aufgefordert, den Vertrag wie bisher zu erfüllen. Dies wurde von ihm abgelehnt. Mit Aufnahme der Vertragsarztpraxis außerhalb des Klinikgeländes kündigte die Klinik den zwischen ihr und dem Chefarzt bestehenden Vertrag fristlos aus wichtigem Grund. Der Chefarzt war nun der Meinung, dass er aufgrund dieser Kündigung wieder zu $100 \%$ Arbeitnehmer des Klinikums sei und forderte die sich hieraus ergebende zweite Hälfte seiner Chefarztvergütung. Das Krankenhaus wollte den Konflikt mit dem Chefarzt nicht eskalieren lassen. Dieser hatte die Qualität der ihm unterstellten Mitarbeiter des Instituts für Pathologie massiv bemängelt. Der Chefarzt wurde daher zunächst gebeten, seine in erheblichem Umfang aufgelaufenen Urlaubsansprüche zu nehmen. Während seines Urlaubs gingen von einem Tag auf den anderen keine Einsendungen entnommener Gewebeproben der umliegenden Kliniken als externe Einsender am Institut für Pathologie der Klinik mehr ein.

\section{Chefarzt warb Einsender ab}

Die Klinik stellte sodann fest, dass der Chefarzt die verantwortlichen Ärzte der umliegenden Kliniken - ohne, dass deren Geschäftsführung davon Kenntnis hatte -, die bislang die entnommenen Proben dem Institut für Pathologie der Klinik zur Begutachtung übersandt hatten, über die seiner Auffassung nach unhaltbaren $\mathrm{Zu}$ stände im Klinikum informiert und veranlasst hatte, alle zukünftig entnommenen Gewebeproben stationärer Patienten nicht mehr im pathologischen Institut der Klink, sondern in seiner Vertragsarzt- praxis gegen Honorar untersuchen zu lassen. Nachdem das Krankenhaus diesen Sachverhalt festgestellt und mit den jeweiligen Geschäftsführern der Kliniken gesprochen hatte, wurde diese Praxis wieder rückgängig gemacht. Die stationär bei externen Kliniken entnommenen Proben wurden sodann wieder dem Institut für Pathologie des Klinikums zur Untersuchung eingesandt.

\section{Klinik kündigte fristlos}

Die Klinik nahm diesen Sachverhalt jedoch zum Anlass, das Arbeitsverhältnis mit dem Chefarzt wegen eines massiven Verstoßes gegen seine arbeitsvertragliche Verpflichtung, seinem Arbeitgeber keinen Wettbewerb zu machen, aufzulösen. Sie kündigte das seit über 20 Jahren bestehende Arbeitsverhältnis fristlos aus wichtigem Grund, ohne den Chefarzt vorher abgemahnt zu haben. Auch die Tatsache, dass das Arbeitsverhältnis 8 Monate später wegen des Renteneintritts des Chefarztes ohnehin geendet hätte, wurde nicht berücksichtigt. Der Chefarzt erhob Kündigungsschutzklage, weil er die Auffassung vertrat, dass er sich nicht vertragswidrig verhalten habe. Außerdem meinte er, dass die Kündigung des $\mathrm{Ar}$ beitsverhältnisses 8 Monate vor Renteneintritt unverhältnismäßig sei und die Klinik vorher hätte zumindest abmahnen müssen.

\section{Die Entscheidung}

In erster Instanz gewann der Chefarzt den Prozess in vollem Umfang. Das Landesarbeitsgericht gab dagegen der Klinik im Rahmen der Berufung durch rechtskräftiges Urteil vom 17.10.2012 (Az.: 20 Sa 94/11) Recht. Ein Arbeitsverhältnis kann aus wichtigem Grund ohne Einhaltung einer Kündigungsfrist gekündigt werden, wenn Tatsachen vorliegen, aufgrund derer dem Kündigenden unter Berücksichtigung aller Umstände des Einzelfalls und unter Abwägung der Interessen beider Vertragsteile die Fortsetzung des Arbeitsverhältnisses selbst bis zum Ablauf der Kündigungsfrist nicht zugemutet werden kann (§ 626 Abs. 1 BGB). Dabei vollzieht sich die erforderliche Prüfung, ob ein gegebener Lebenssachverhalt einen wichti- 
gen Grund in diesem Sinne darstellt, zweistufig. Zunächst ist zu prüfen, ob ein bestimmter Sachverhalt ohne die besonderen Umstände des Einzelfalls als wichtiger Kündigungsgrund an sich geeignet ist. Ist dies der Fall, bedarf es der weiteren Prüfung, ob dem Kündigenden die Fortsetzung des Arbeitsverhältnisses unter der Berücksichtigung der konkreten Umstände des Einzelfalls und unter Abwägung der Interessen beider Vertragsteile jedenfalls bis zum Ablauf der Kündigungsfrist zumutbar ist oder nicht.

\section{Arbeitnehmer ist während Arbeitsverhältnis Konkurrenz- tätigkeit untersagt}

Nach Auffassung des LAG erwies sich unter diesen Prämissen die fristlose Kündigung des Krankenhauses aufgrund massiver Wettbewerbsverstöße des Klägers als wirksam. Zunächst stellten die Richter fest, dass während des rechtlichen Bestehens eines Arbeitsverhältnisses einem Arbeitnehmer grundsätzlich jede Konkurrenztätigkeit zum Nachteil seines Arbeitgebers untersagt ist. Dies gelte auch dann, wenn der Arbeitsvertrag keine Regelungen hierüber enthielte. Der Arbeitgeber soll vor Wettbewerbshandlungen eines Arbeitsnehmers geschützt werden. Ist allerdings ein nachvertragliches Wettbewerbsverbot nicht vereinbart, ist es dem Arbeitnehmer schon vor Beendigung des Arbeitsverhältnisses für die Zeit nach seinem Ausscheiden erlaubt, die Gründung eines eigenen Unternehmens oder den Wechsel zu einem Konkurrenzunternehmen vorzubereiten. Verboten sei lediglich die Aufnahme einer werbenden Tätigkeit, z.B. durch Vermittlung von Konkurrenzgeschäften oder aktives Abwerben von Kunden. Bloße Vorbereitungshandlungen, die in die Interessen des Arbeitgebers nicht unmittelbar eingreifen, erfüllen diese Voraussetzung nach Auffassung des Gerichts nicht.

Die Richter stellten fest, dass die Verletzung eines für die Dauer des Arbeitsverhältnisses bestehenden Wettbewerbsverbots an sich einen wichtigen Grund für eine außerordentliche Kündigung darstellen könne. Gegen dieses vertragsbegleitende Wettbewerbsverbot habe der Chefarzt verstoßen. Der Chefarzt habe übersehen, dass die rechtliche Grundlage der Nebentätigkeit weggefallen sei, er die Nebentätigkeit aber gleichwohl weiter ausgeübt habe. Außerdem habe er offensichtlich und absprachewidrig Aufträge von Kunden des Krankenhauses auf seine Vertragsarztpraxis umgeleitet bzw. dies versucht. Der Kläger habe mit der vertragswidrig betriebenen Praxis pathologische Labor- bzw. Befundleistungen am Markt erbracht. Er habe jedenfalls versucht, über den ambulanten Bereich hinaus den sogenannten stationären Bereich für seine Vertragsarztpraxis zu gewinnen. Dieses Verhalten sei im Zusammenhang mit seiner Stellung als Leiter des pathologischen Instituts der Klinik nicht zu vereinbaren. Hierin sahen die Richter einen Verstoß gegen seine Vertragspflichten in besonders massiver Weise. Der Kläger habe nicht nur ohne Beteiligung des Krankenhauses an den Einnahmen und vertragswidrig in eigenen Praxisräumen eine Vertragsarztpraxis betrieben. $\mathrm{Er}$ habe auch versucht, das Betätigungsfeld der Vertragsarztpraxis zum Nachteil der Klinik zu erweitern.

Eine Fortsetzung des Arbeitsverhältnisses bis zum Ablauf der Frist für eine ordentliche Kündigungsfrist sei der Klinik nicht zuzumuten gewesen. Zwar ließen sich bei der Interessensabwägung zu berücksichtigende Umstände nicht abschließend und für alle Fälle einheitlich festlegen. Gehe es um die Beurteilung rechtswidrigen schuldhaften Verhaltens des Arbeitnehmers, seien aber stets die beanstandungsfreie Dauer des Arbeitsverhältnisses, das Gewicht und die nachteiligen Auswirkungen einer Vertragspflichtverletzung, eine mögliche Wiederholungsgefahr und der Grad des Verschuldens des Arbeitnehmers zu berücksichtigen. Nach Auffassung der Richter überwog das Beendigungsinteresse der Klinik. Zwar sei die Beendigung des Arbeitsverhältnisses absehbar gewesen. Der Kläger hätte jedoch nicht nur die Absicht bekundet, auch danach in irgendeiner Weise im Fachgebiet tätig zu bleiben. Er habe auch durch die Gründung einer eigenen Praxis außerhalb der Klinikräume der Klinik verdeutlicht, wie er gedachte, künftig auf seinem Fachgebiet tätig zu sein. Damit bestand für die Beklagte die ganz erhebliche Gefahr, dass der Chefarzt bis zu seinem Ausscheiden aus ihren Diensten versuchen würde, sich „Marktanteile zu sichern“. Dabei spiele es keine Rolle, ob die Praxis dauerhaft im Namen des Chefarztes oder im Namen eines anderen - beispielsweise seines Sohnes - weitergeführt werden sollte. Denn dem Arbeitnehmer ist aufgrund des Wettbewerbsverbots nicht nureine Konkurrenztätigkeit im eigenen Namen und Interesse untersagt, sondern ihm ist es gleichfalls nicht gestattet, einem Arbeitskollegen bei einer konkurrierenden Tätigkeit zu helfen oder einen Wettbewerber des Arbeitsgebers zu unterstützen. Über zulässige Vorbereitungshandlungen für die Zeit nach seinem Ausscheiden gehe das Verhalten des Chefarztes hinaus.

\section{Abmahnung war entbehrlich}

Eine Abmahnung war nicht erforderlich. Eine Abmahnung ist dann entbehrlich, so die Richter, wenn die Pflichtverletzung dem Arbeitnehmer ohne weiteres erkennbar ist und eine Hinnahme durch den Arbeitgeber offensichtlich ausgeschlossen ist oder eine Verhaltensänderung selbst nach einer Abmahnung nicht zu erwarten ist. Dies sei vorliegend der Fall. Der Kläger könne nicht mit vertretbaren Gründen davon ausgehen, die Klinik werde sein Verhalten hinnehmen und jedenfalls nicht zum Anlass für den Ausspruch einer Kündigung nehmen.

\section{Fazit}

Angestellte Chefärzte sollten im Auge behalten, dass Verletzungen ihrer vertraglichen Pflichten aus Nebenabreden zum Arbeitsvertrag bzw. Nutzungs- und Kooperationsverträgen zu fristlosen Kündigungen des Arbeitsverhältnisses führen können. Sie riskieren also ggf. nicht nur die Nebenabreden, sondern ihr gesamtes Arbeitsverhältnis. Da es sich letztlich immer um Einzelfallentscheidungen handelt, verbietet sich eine pauschale Beurteilung. Festgehalten werden kann aber jedoch, dass ein Angestellter seinem Arbeitgeber während des Arbeitsverhältnisses keinen Wettbewerb machen darf. Die Situation wird sicherlich häufig dann von Bedeutung, wenn der Angestelltenstatus aufgrund Erreichens der Altersgrenze in näherer Zukunft endet und der Chefarzt nach seinem Ausscheiden ambulant noch weiter tätig sein will. Hier ist es ratsam, mit „offenen Karten“ zu spielen und sich zumindest über die eigene rechtliche Position im Klaren zu sein.

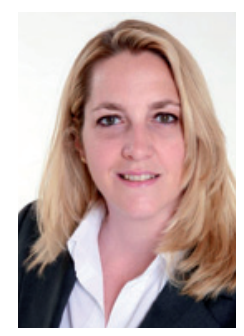

Korrespondenz Dr. iur. Isabel Häser Rechtsanwältin ECOVIS Lüdemann Wildfeuer \& Partner Sonnenstr. 9 80331 München www.ecovis.com 